\title{
Optical Design for the Composite InfraRed Spectrometer Lite (CIRS-Lite)
}

\author{
Bert A. Pasquale ${ }^{\mathrm{a}}$, John Brasunas ${ }^{\mathrm{a}}$, John Hagopian ${ }^{\mathrm{a}}$, Qian Gong ${ }^{\mathrm{a}}$, William Mamakos ${ }^{\mathrm{b}}$, Melissa \\ Edgerton $^{\mathrm{a}}$, Vincent Bly ${ }^{\mathrm{a}}$ \\ ${ }^{a}$ NASA/Goddard Space Flight Center, Greenbelt, MD 20771; \\ ${ }^{\mathrm{b}}$ Design Interface Inc., Finksburg, MD 21048
}

\begin{abstract}
Following up on Cassini/CIRS, we are building the next-generation Composite InfraRed Spectrometer for deep-space planetary exploration. CIRS-Lite combines Mid \& Far-IR channels into a single instrument with $4 \mathrm{x}$ the spectral sensitivity of CIRS. Here we discuss the instrument optical design, the design process, and the system performance.
\end{abstract}

Keywords: Optical Design, Optical Fabrication, Instruments, Spectroscopy, Fourier Transform, Silicon Optics, Planetary Science, Infrared

\section{INTRODUCTION}

The Cassini/CIRS Composite InfraRed Spectrometer has been operating beyond its designed lifetime in orbit around Saturn, and has returned a wealth of information about the gas giant, its rings and its moons. CIRS-Lite directly builds on that heritage [1] while simplifying the design to combine both the Mid- and Far-Infrared channels into the same optical path. The anticipated return to Saturn/Titan or Jupiter would be a direct application of the design, which is currently being built as an engineering unit. Many applications of this type of design have been considered by the planetary sciences community. [2] The CIRS-Lite design is shown in Figure 1, below.

Operating at $140 \mathrm{~K}^{\circ}$, CIRS-Lite uses a Michelson interferometer to modulate the signal for each pixel field. A doublepass scanning corner cube provides $0.1 \mathrm{~cm}-1$ spectral resolution, retrieved from the signal via Fourier Transform. The total weight of the instrument was originally designed to be kept to under $20 \mathrm{~kg}$, though several design implementations would allow it to be made much more lightweight.

\section{OPTICAL LAYOUT}

While the basic layout of the design mimics much of the CIRS MIR channel, there are several differences for packaging and to accommodate the FIR waveband out to 333 microns. The basic system consists of:

- 6-inch Aperture Cassegrain Telescope

- Relay (Fore) Optics, including a field mirror and collimator

- Interferometer, with double-pass scanning corner cube

- Focus Optics

- Focal Plane Array, with concentrators.

Figure 1 shows the layout of CIRS-Lite optical system. 


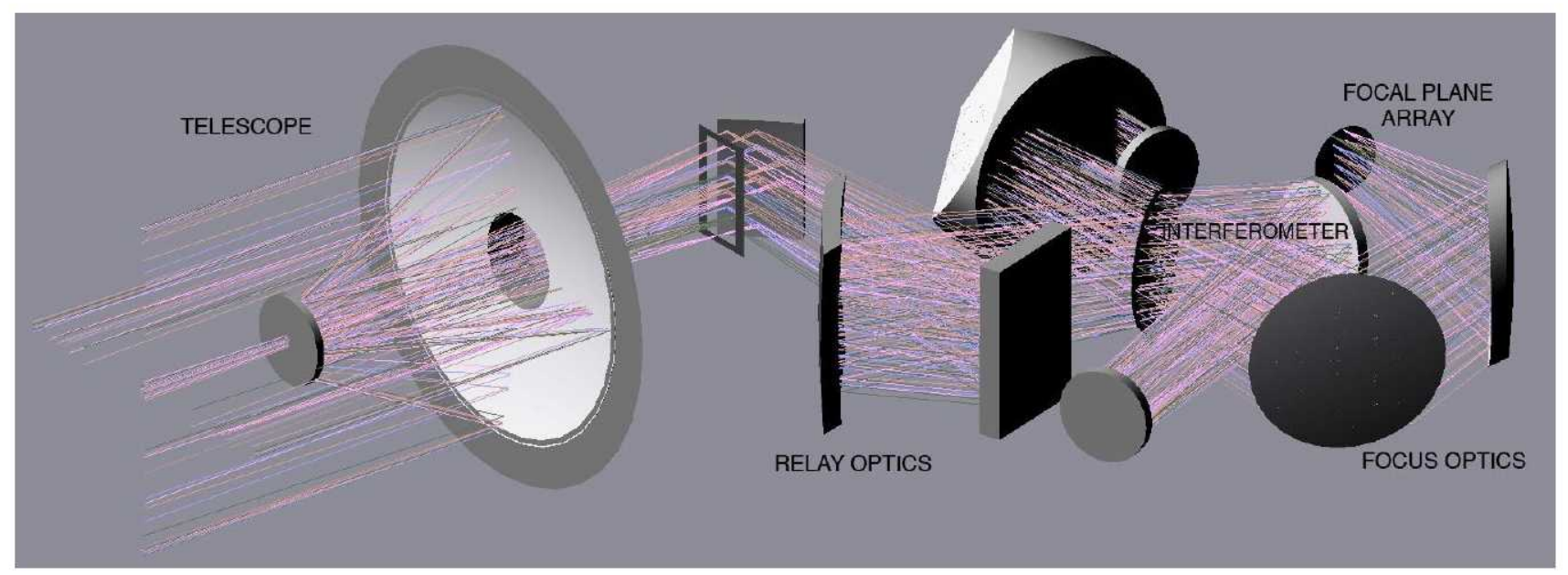

Figure 1. The CIRS-Lite optical System.

\subsection{Fields of View}

The MIR channel consists of two $1 \times 28200 \mu \mathrm{m}$ pixel $\mathrm{HgCdTe}$ arrays, sensitive to 7.1-9.1 $\mu \mathrm{m}$ and 9.1-16.7 $\mu \mathrm{m}$, respectively. Each Mid-Infrared pixel covers a $1.5 \times 1.5 \mathrm{mrad}$ FOV. The total allocated field height is 2.4 degrees by 1 degree. The FIR channel consists of one $1 \mathrm{x} 4$ bolometer array, sensitive to $333 \mu \mathrm{m}$. Each pixel is $4.3 \mathrm{mrad}$ wide. The layout of the three channels is shown in Figure 2.

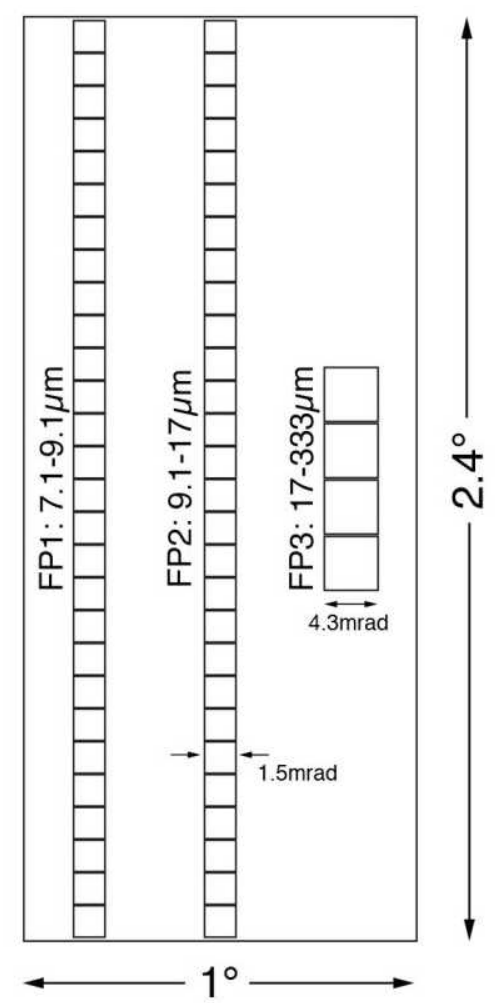

Figure 2. The CIRS-Lite Field of View allocation map 


\subsection{Telescope}

The telescope (shown in Figure 3) is $150 \mathrm{~mm}$ in diameter with an $\mathrm{f} / 7$ focus. The secondary mirror is supported by struts off of the baffle/sunshade. The telescope pieces are fabricated from single-crystal silicon, and polished with EDM (electro-discharge machining) to a super-smooth figure.

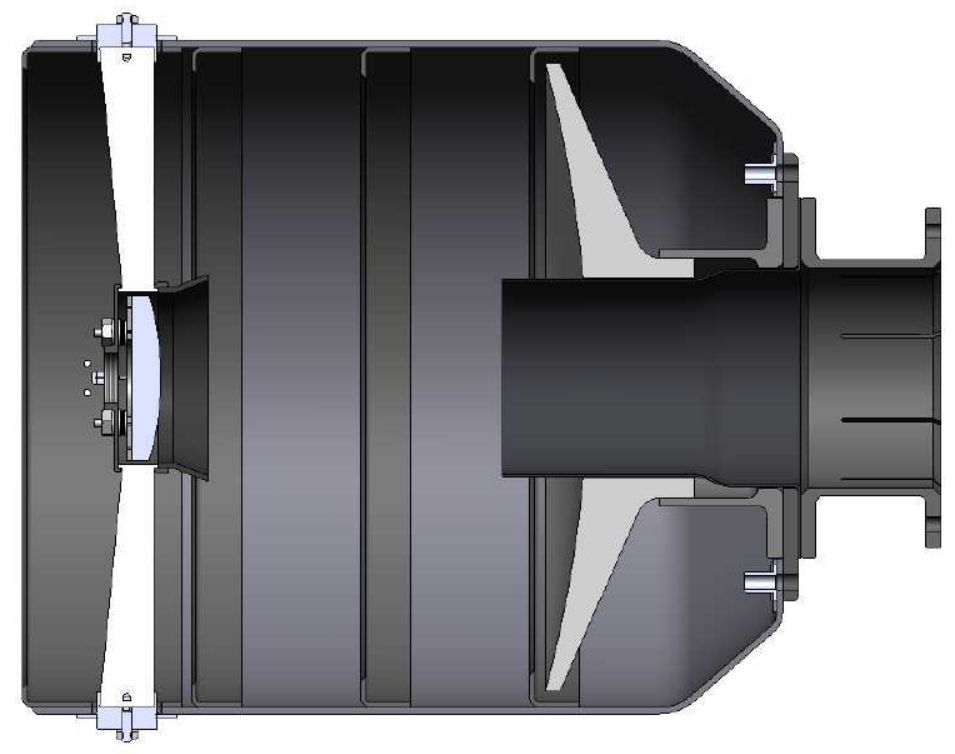

Figure 3. CIRS-Lite single-crystal silicon telescope showing the primary, secondary and baffle structure.

An Entrance Aperture Plate (EAP) acts as a stray light component at the telescope focus. The EAP is an oversized baffle, not an actual field stop. It is located where the edges can cleanly clip around the fields without taking away from in-field radiation. This is an important stray light component in that it limits extraneous light from entering the aft optical system. The Entrance Aperture Plate is seen in Figure 4 as a cut-out rectangle, and would be fully enclosed to not allow light to pass around it. The EAP and the optics bench will be cooled to $170^{\circ} \mathrm{K}$.

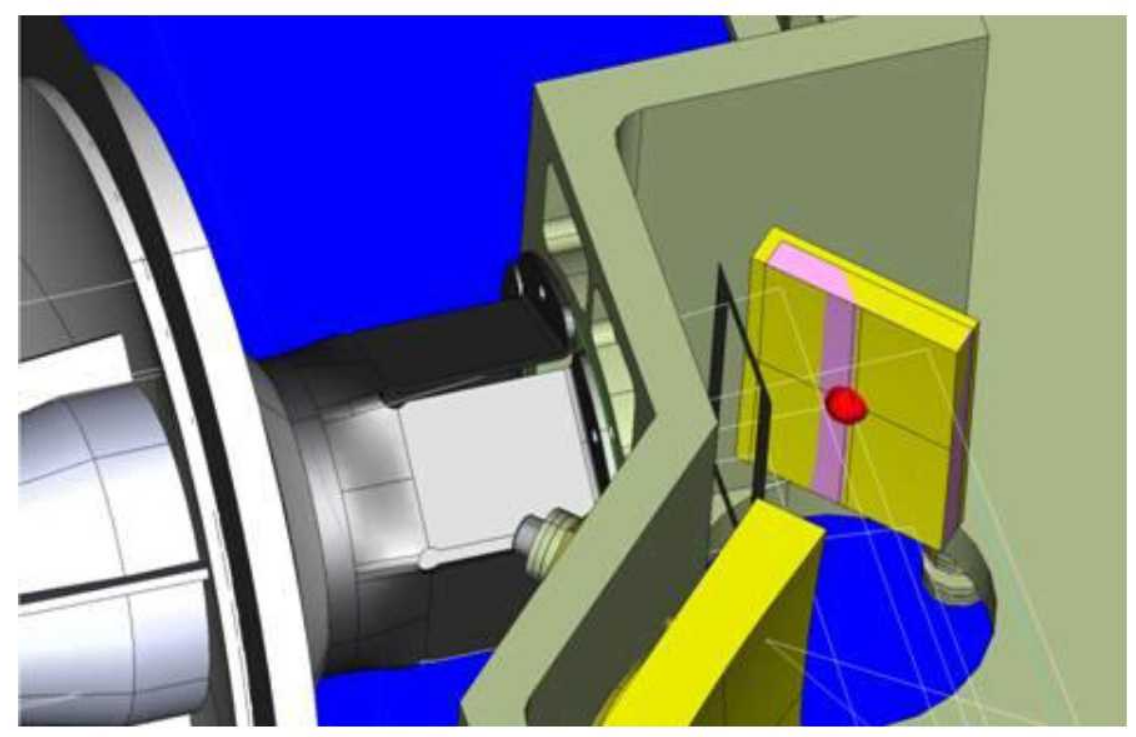

Figure 4. CIRS-Lite Entrance Aperture Plate (outline shown.) 


\subsection{Relay optics}

An anamorphic fold mirror immediately after focus (also seen in Figure 4) allows for the pupil to be accurately placed and well formed later within the interferometer. An additional fold mirror and off-axis Parabola collimate the beam and relay it to the interferometer. This and the entire optical bench is shown in Figure 5.

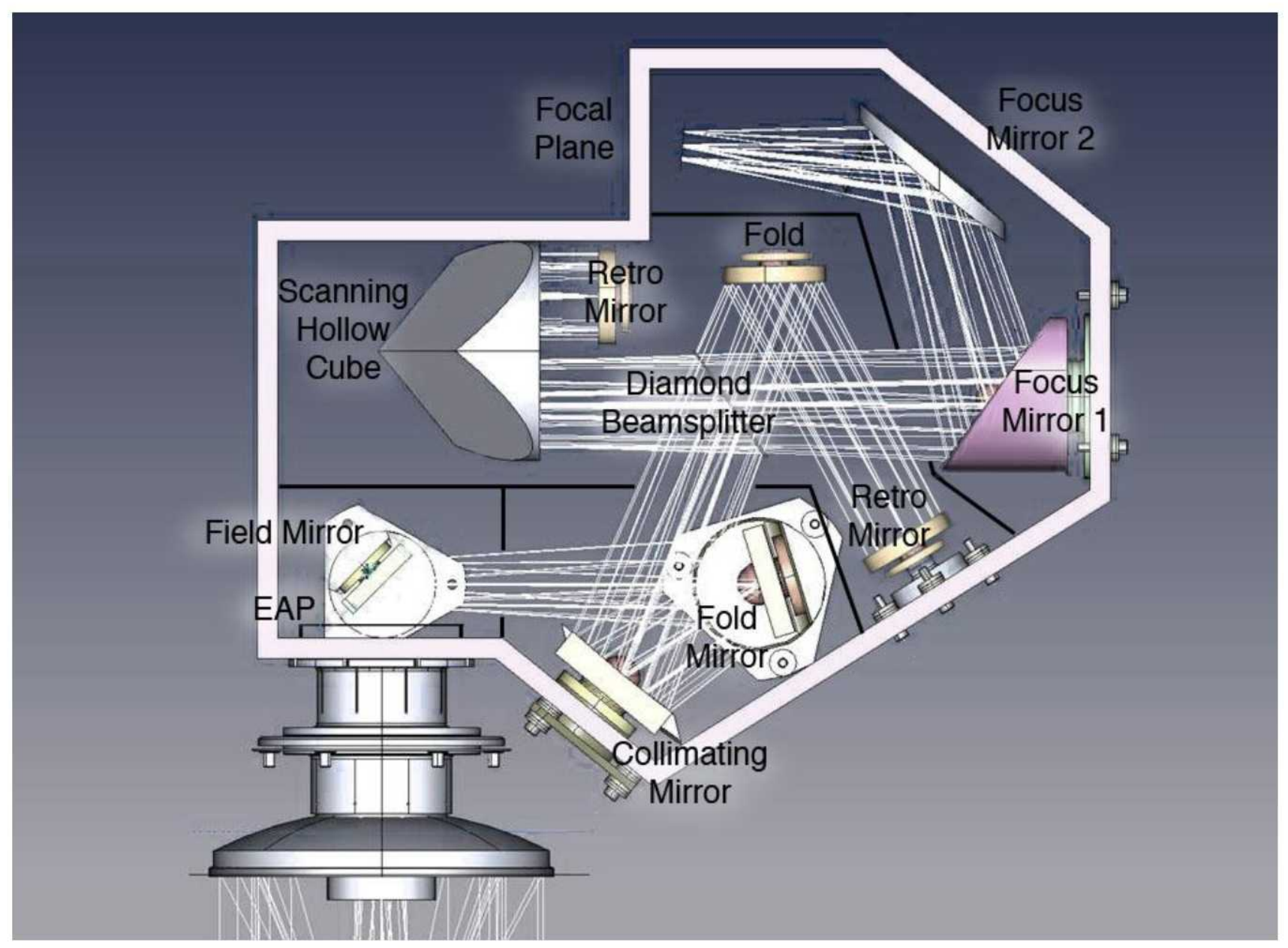

Figure 5. The CIRS-Lite optical bench. bench, including components, ray bundles, and baffle layout

\subsection{Interferometer}

The interferometer uses a synthetic diamond beamsplitter. The beamsplitter has a thickness of about $1 \mathrm{~mm}$ and a slight wedge for ghosting control and a metallic thin film on its reflective surface. No compensator plate is needed.

Within the interferometer, the pupil is formed at the retroreflecting mirrors in each arm. The beam passing through the beamsplitter forms its pupil on the fixed optical path mirror, while the reflected beam is directed through a doublepassed corner cube. It is not until the beam exits the cube, laterally displaced to be beside itself, that the pupil is formed at the retroreflecting mirror. The nominal zero-path difference position of the cube is biased towards the mirror, with most the scan path length moving away from the mirror. 


\subsection{Focus Optics}

The focus optics are based on a Crossed Dragone two-mirror design. [3] Together, they form an image in-plane to the rest of the instrument, allowing the detector package to be located outside the optical bench. The focal plane is rotated about the axis normal to the optical bench plane, but maintains its vertical orientation and no clocking.

\subsection{Focal Plane \& Concentrators}

The image is formed at the opening of Concentrators, which will provide up to $8 \mathrm{x}$ concentration in the MIR and up to $5 \mathrm{x}$ concentration in the FIR.

Because the concentrator design is different, the FIR detectors are not co-planer with the MIR. Each pixel is physically separated from its neighbor due to the concentrator cone, making the image plane about $40 \mathrm{~mm}$ long.

\section{DESIGN PROCESS}

Optimizing the CIRS-Lite system begins with the telescope, moves to the relay collimation, into the interferometer, back to the relay, and then to the focus design. Of course, may iterations of design options may and in fact have occurred.

\subsection{Telescope}

The telescope (See figure 6) was designed to be compact, with an $\mathrm{f} / 1$ mirror. This is reasonable, noting that the original CIRS mirror was $.5 \mathrm{~m}$ in diameter and $\mathrm{f} / 0.7$. Optimizing to an $\mathrm{f} / 7$ beam kept the image size and hence the cumulative fields' ray bundles a consistent width along the span from the Secondary mirror through the Primary mirror hole and to the Entrance Aperture Plate. This provided adequate image quality and allowed for the baffling tube to extend well into the telescope. This will be revisited after checking for vignetting in the Interferometer.

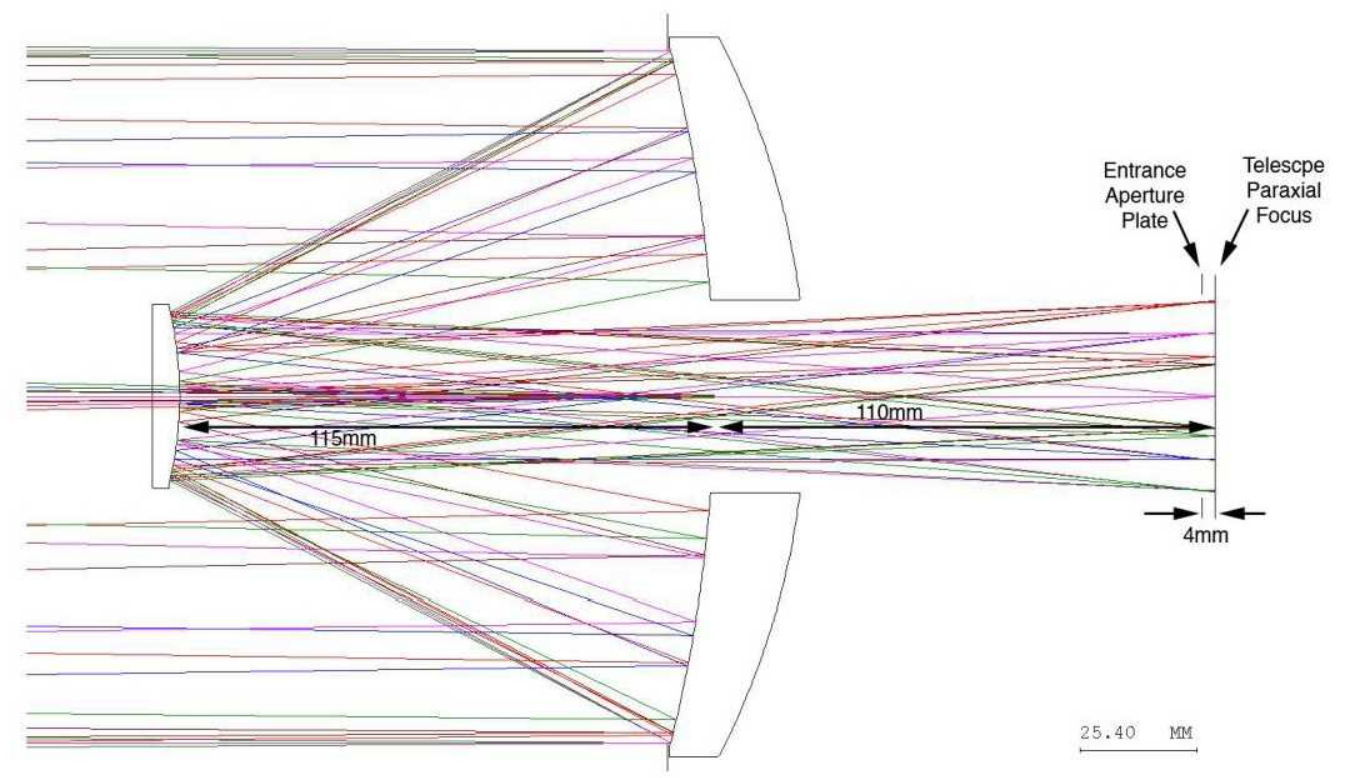

Figure 6. The CIRS-Lite Telescope showing the Entrance Aperture Plate location 4mm before paraxial focus. 


\subsection{Relay Optics}

The relay optics were designed to accomplish two tasks: Collimate the light beam and set the pupil position. The collimation is accomplished by an off-axis asphere. Initially we used a parabola, then moved to a generalized anamorphic asphere, allowing for improved collimation quality. The collimator is sized to create a $35 \mathrm{~mm}$ diameter beam for the interferometer. This results in an angular magnification of $\mathrm{x} 4.3$ from the $150 \mathrm{~mm}$ Telescope aperture.

Power is then applied to the field mirror to control the shaping and placement of the pupil at the estimated location of the Michelson retroreflectors. (This is done iteratively as the position of the retro mirror is refined during the interferometer design. This does not usually necessitate a re-optimization of the collimator, as the field mirror is very close to the telescope image and does not reshape the wavefront.) The angles of the fold mirror are used to align the collimated beam to an appropriate angle and location for the intended interferometer layout. In this case, a $34^{\circ}$ reflectance angle was used for the collimator, and the output beam was angled $30^{\circ}$ to the original telescope optical axis.

\subsection{Interferometer}

The interferometer is a Michelson Interferometer with a double-pass scanning corner cube providing the signal modulation. The $30^{\circ}$ beam from the collimator is aligned to the beamsplitter also at $30^{\circ}$, so the reflection is not orthogonal to the original telescope axis, along with the scan mechanism axis.

The beamsplitter is a synthetic diamond, with a $60 \mathrm{~mm}$ diameter. Because of field divergence and the 4.3 angular magnification, off-axis fields' beams are vignetted by the beamsplitter. This is minimized by holding the diamond with only a $2 \mathrm{~mm}$ loss of clear aperture. Vignetting will need to be carefully calibrated.

We attempt to minimize the distance from the beamsplitter to the retro mirror. This is limited by the physical size of the corner cube, which is made large enough to provide lateral displacement of the beam to come out beside itself. In this case, a 4" aperture cube sufficed. The total optical path length from the beamsplitter to the retroreflecting mirror was $\sim 225 \mathrm{~mm}$. The corner cube is a hollow mirror cube, with the faces bonded to each other to create "seamless" interfaces. This is the same technology developed for the CIRS corner cubes.

We now know the effective distance to the retroreflector, and can adjust the power in X \& Y on the Field Mirror to form a tight pupil on the retro mirror position. By placing the pupil there, vignetting is minimized and made symmetric as the beam passes through the beamsplitter coming in and leaving the interferometer.

\subsection{Vignetting Effects on the Design}

The design of the aft optics and interferometer did not initially consider vignetting effects in ray bundle clearances. Hence, the mirror sizes and beam clearances may be oversized for the interferometer. By clipping the rays that do not pass the beamsplitter, we can now do two things: Check clearances in the telescope, and redesign the relay optics to have tighter integration to the interferometer.

Now that the vignetting is set, the final beam clearances can be checked and verified there is no interference with the structures. Baffles can be finalized for their clearances, and stray light analysis performed. Figure 7 shows the footprint of the vignetted beams moving through the telescope tube, from the Secondary Mirror, through the Primary Mirror central hole, to the Entrance Aperture Plate. It can be clearly seen how vignetting affects the off-axis beam profiles. 

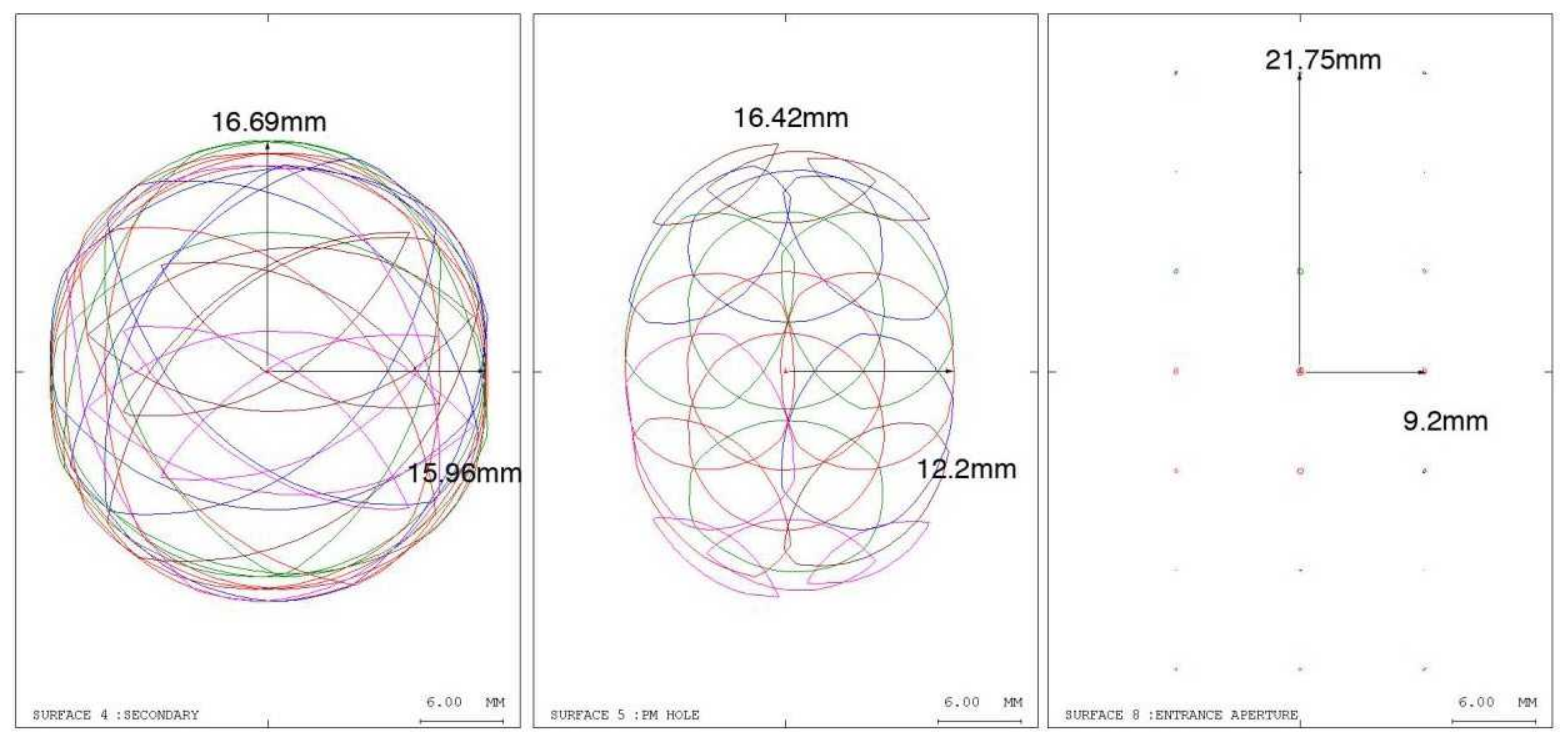

Figure 7. CIRS-Lite Beam Sizes in the Telescope, showing vignetting effects ( 21 field points.)

Note that the Entrance Aperture Plate is located $4 \mathrm{~mm}$ in front of the telescope's paraxial focus. This is where the points around the edge intersect the curved telescope image surface.

\subsection{Focus Optics}

Rather than constrain the parent vertex of the focus mirrors, constraints were placed on the location of the chef ray intersection points in global space. This allowed the mirrors to explore design space including anamorphic and higherorder aspheric terms with tilts. (Such surfaces are easily made on modern diamond-turning machines.) By allowing the parent vertices to move freely, a solution was found that uses skew and rotated cutouts of the parent, as shown in Figure 8. The increase in performance was about 2.5 -fold from the base design.

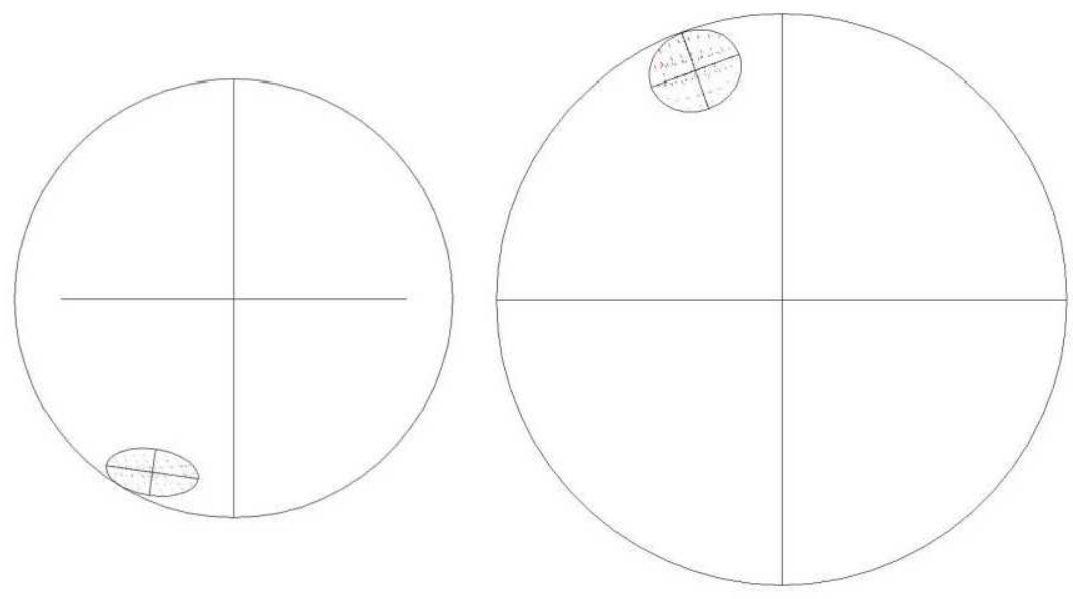

Figure 8. Optical Parents of the two Focus Mirrors, and the clear aperture cutouts. 


\subsection{Focal Plane}

To achieve platescale requirements for the $200 \mu \mathrm{m}$ detectors, the focus optics would have to be $\sim \mathrm{f} / 1$. The coarse pixel FOV allows us to rather use a slow focus beam and an array of concentrators. This allows us to create a layout that both corrects for field distortion, and by using different concentrator powers adjust the platescale for the FIR.

\section{PERFORMANCE}

The image plane platescale before the pixel concentrators provides approximately $1.2 \mathrm{~mm}$ per pixel. The geometrical RMS spot size is about $1 / 10$ the pixel IFOV. The MIR channels are RMS $60-90 \mu \mathrm{m}$ and the FIR is RMS $160-180 \mu \mathrm{m}$ Figure 9 shows the spots to scale with the geometrical FOV. Note that the fields do have a "smile" distortion and keystone. This can be compensated for in the fabrication of the concentrator \& detector array.

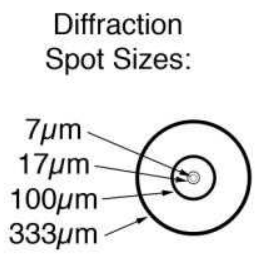

$10 \mathrm{~mm}$

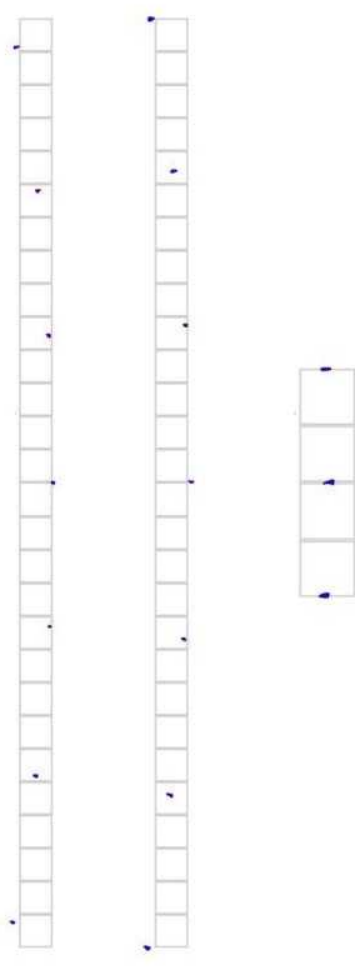

Figure 9. Geometrical Spot Sizes Across the Image Plane with diffraction sizes shown.

At the maximum wavelength in the MIR $(17 \mu \mathrm{m})$ diffraction increases the spot size to $180 \mathrm{um}(80 \% \mathrm{EE}$.) The IOFV of the each pixel retains $94-99 \%$ of the original energy at the $1.6 \mathrm{~mm}$ opening to the concentrator cones. See Figure 10 below. 


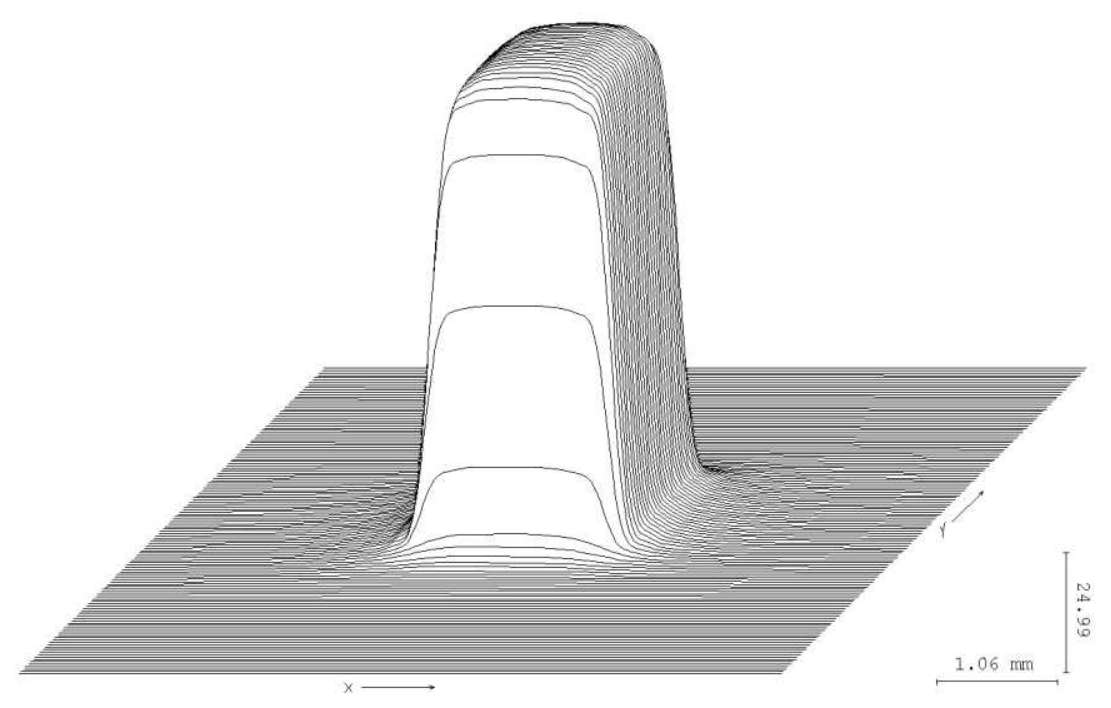

Figure 10. IFOV of a Full-Field MIR Pixel at Maximum Wavelength.

While the FIR image plane is larger $(\sim 1.7 \mathrm{~mm})$, the spot size at the maximum wavelength $(333 \mu \mathrm{m})$ in the FIR approaches $4 \mathrm{~mm}$. IVOF efficiencies vary with longer wavelengths from $97 \%$ down to $86 \%$. (See Figure 1la/b.)
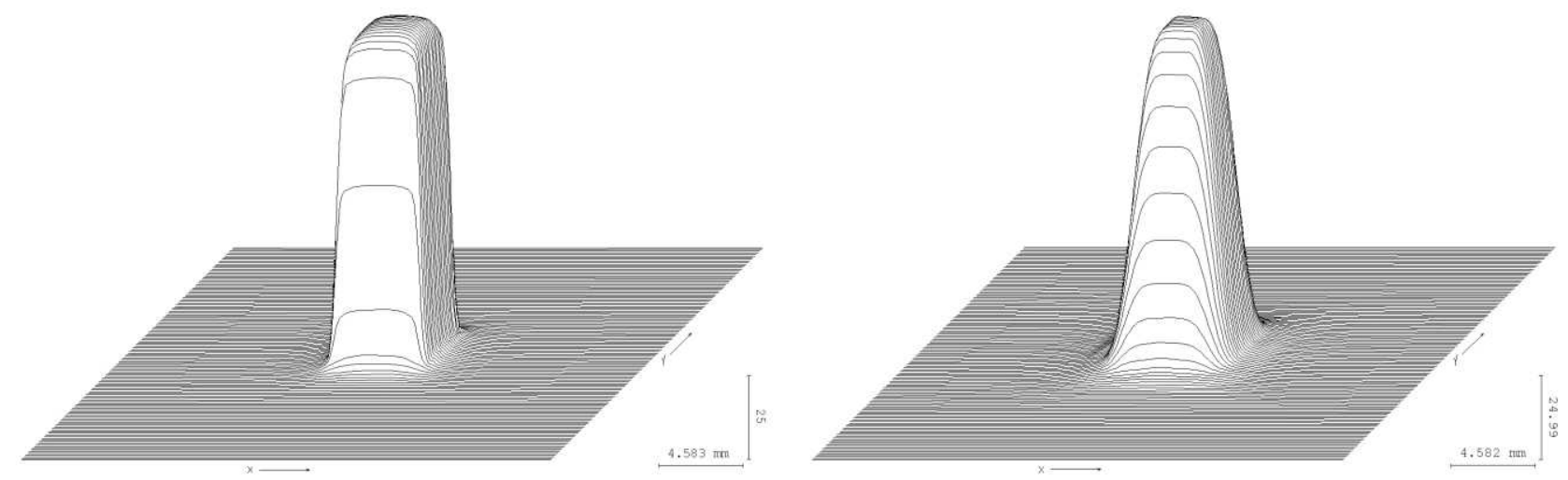

Figure $11 \mathrm{a} / \mathrm{b}$. IFOV of a Full-Field FIR Pixel at $100 \mu \mathrm{m}$ and $333 \mu \mathrm{m}$.

\section{REFERENCES}

[1] Peter Maymon, "Optical design of the Composite InfraRed Spectrometer (CIRS) for the Cassini mission," SPIE Proc. 1945, 100-111 (1993).

[2] John Brasaunus, "Future developments in planetary Fourier transform spectroscopy at NASA's Goddard Space Flight Center," SPIE Proceedings Vol. 5660, Instruments, Science, and Methods for Geospace and Planetary Remote Sensing, 241-250, (2004).

[3] Christophe Granet, "Designing classical Dragonian offset dual-reflector antennas from combinations of prescribed geometric parameters," Antennas and Propagation Magazine, Vol. 45 No. 6, 100-106 (2001). 